\title{
Balkanologie
}

Balkanologie Revue d'études pluridisciplinaires

Vol. II, nº 1 | 1998

Volume II Numéro 1

\section{La genèse de l'émigration politique serbe et croate}

\section{Marina Glamočak}

\section{OpenEdition}

Journals

Édition électronique

URL : http://journals.openedition.org/balkanologie/239

DOI : 10.4000/balkanologie.239

ISSN : 1965-0582

\section{Éditeur}

Association française d'études sur les Balkans (Afebalk)

\section{Édition imprimée}

Date de publication : 1 juillet 1998

ISSN : 1279-7952

\section{Référence électronique}

Marina Glamočak, "La genèse de l'émigration politique serbe et croate », Balkanologie [En ligne], Vol. II, $n^{\circ} 1$ | 1998, mis en ligne le 30 novembre 2007, consulté le 17 décembre 2020. URL : http://

journals.openedition.org/balkanologie/239; DOI : https://doi.org/10.4000/balkanologie.239

Ce document a été généré automatiquement le 17 décembre 2020.

(C) Tous droits réservés 


\title{
La genèse de l'émigration politique serbe et croate
}

\author{
Marina Glamočak
}

1 L'idée de faire une recherche portant sur l'émigration yougoslave, en particulier serbe et croate, a coïncidé avec les changements intervenus en Yougoslavie au début des années 1990, changements qu'on espérait démocratiques. En ce sens, il était intéressant d'analyser les programmes politiques de la diaspora et sa contribution idéologique et politique, soit pour réformer, soit pour détruire la société yougoslave. La guerre qui a éclaté dans ce pays rendait d'autant plus nécessaire, à notre sens, cette étude ${ }^{1}$.

2 Il s'agissait de recenser les facteurs les plus importants pour évaluer les capacités de l'émigration à réagir aux réalités apparues après la chute du communisme. Dans ce but, nous proposons une analyse de la genèse de l'émigration politique serbe et croate pendant la guerre froide. Elle est présentée ici en quatre parties.

3 La première partie porte sur la composition sociale de l'émigration politique, considérée comme l'un des aspects les plus importants pour évaluer les capacités d'une émigration de participer à la vie politique de son pays d'origine. Sa composition sociale regroupe à la fois la structure sociale de l'émigration, son capital idéologique (démocrates, monarchistes, pro-nazis, ...), ainsi que son rôle lors de la Deuxième Guerre mondiale (les groupes armés par exemple). Notre analyse de l'émigration politique croate et serbe n'englobe pas l'émigration communiste d'après 1948 (année de la résolution du Kominform).

4 La deuxième partie s'attachera à montrer que l'instauration des nouveaux régimes communistes dans l'Europe de l'est ne devait pas nécessairement produire une émigration politique. C'est l'appareil répressif légalisé qui a, à la fois, empêché le retour d'une partie des personnes déplacées et produit de nouveaux émigrés.

Dans la troisième partie, nous traiterons de l'émigration politique serbe et croate par rapport au phénomène général d'émigration politique de l'est européen. Celle-ci venant de l'autre côté du "rideau de fer", durant toute la période de la guerre froide, constituait une opposition au communisme allant de l'anticommunisme et du nationalisme au terrorisme. Dans ce texte nous aborderons seulement la question de 
l'anticommunisme et du nationalisme. Nous allons voir, d'un point de vue théorique, que l'émigration politique n'a apporté aucune contribution à l'anticommunisme à la différence du mouvement dissident. Son anticommunisme avait un caractère déclaratif, opposant au régime communiste le libéralisme (économique) et la démocratie (politique). Par contre, son nationalisme était générateur d'une lutte à la fois contre l'État yougoslave et le régime communiste établi. Un passage particulier sera consacré au système idéologique des groupes émigrés.

$6 \mathrm{Vu}$ qu'il est inhérent à la nature de l'émigration politique de chercher des appuis militaires et politiques, nous ferons un historique de l'utilisation de l'émigration politique, plus particulièrement au début de la guerre froide. Dans cette dernière partie de recherche, nous examinerons la particularité du cas yougoslave, qui réside dans l'absence de soutien de la part de l'Occident. La frange extrémiste de l'émigration politique n'avait pas, sur le plan idéologique, une grande importance pour le régime communiste car elle était si divisée, selon l'appartenance nationale, qu'elle ne pouvait en aucune façon être une alternative au régime titiste. Cela d'autant moins qu'après 1948 l'Occident fit de Josip Broz-Tito son dissident politique préféré et lui assura qu'il ne soutiendrait pas les groupes nationalistes et par la même une dissolution de la Yougoslavie. Les groupes démocratiques des émigrés politiques, en revanche, constituaient un véritable danger pour le pouvoir en Yougoslavie car ils proposaient des réformes et la démocratisation de la société yougoslave, mais, à l'instar de l'opposition existant sur l'espace ex-yougoslave, ils ne furent pas soutenus.

\section{La composition sociale de l'émigration politique}

7 Une des questions cruciales qui se pose pour toute émigration politique est de savoir si elle garde une continuité avec les idées politiques qui jouaient un rôle dans son pays avant qu'elle ne le quitte. Une autre question, tout aussi importante, consiste à savoir si les caractéristiques de l'émigration pourraient être prises pour un indicateur relativement fiable du paysage politique du pays, concrètement de la Yougoslavie, si le monopole du Parti communiste n'y avait introduit une discontinuité.

Le degré de représentativité politique de l'émigration et le degré de continuité relative de la pensée politique dépendent du caractère soit élitiste, soit de masse de cette émigration. Par conséquent, l'émigration yougoslave est conditionnée par sa composition sociale, la grande différence entre les émigrations politiques croate et serbe provenant de leur composition sociale ainsi que de leur organisation.

\section{L'émigration serbe}

9 À la fin de la Deuxième Guerre mondiale, l'émigration politique serbe était plus importante que l'émigration croate car, en plus de formations militaires qui quittaient le pays, près de 300000 Serbes, prisonniers de guerre ou travailleurs déportés, se trouvaient déjà en Allemagne. Outre son caractère massif, l'émigration serbe de la période d'après-guerre est caractérisée par la sur-représentation des cadres militaires. Et cela bien que des Serbes et Slovènes en armes, qui s'étaient rendus aux Alliés, plus précisément aux Britanniques, aient été livrés aux partisans et massacrés dans les bois de Kočevje ${ }^{2}$. Leur nombre s'élèverait à 14000 personnes, selon Kneževićs ${ }^{3}$. 

bataillons tchetniks originaires de la Lika, de la Banija et du Kordun, et de Bosnie, parvinrent pratiquement intacts à sortir du pays avec leurs familles. En effet, les bataillons de l'Armée yougoslave dans la patrie sous le commandement du général Mihailovićt, qui étaient stationnés en Serbie au cours de la guerre, lorsqu'ils tentaient de quitter le pays, furent décimés lors de combats contre les partisans et les oustachis en Bosnie ainsi que par le typhus.

11 L'émigration serbe est donc plus largement issue de la diaspora serbe installée au-delà de la Drina, en Bosnie, que de la Serbie même.

Si les Serbes de Serbie constituaient les deux tiers du peuple serbe, ils formaient à peine le quart de l'émigration ; les Serbes, originaires d'autres régions que la Serbie, étaient les plus nombreux. Mon intention n'est pas de les sous-estimer, (...) mais par rapport aux Serbes de Serbie ils ont un faible niveau de conscience politique. Cela se voit, aujourd'hui, du fait que les tendances extrémistes existant dans la société serbe proviennent plus de Bosnie, de Croatie, du Monténégro aussi, que de Serbie même. Le mouvement extrémiste en Serbie ne peut pas, même à l'heure actuelle, constituer une force importante. ${ }^{5}$

L'émigration politique serbe d'alors n'était pas à proprement parler composée d'éléments politiques. Il est vrai qu'elle était anti-communiste, mais cet anticommunisme avait plus un caractère émotionnel que politique. Avant la guerre, elle n'avait eu aucun contact avec les communistes car le Parti communiste avait été interdit et de ce fait ne participait pas à la vie politique yougoslave. L'émigration serbe était donc contre le communisme, non pas à cause de l'idéologie qu'elle ne connaissait guère, mais avant tout parce que le nouveau régime avait destitué le roi.

La vague d'émigration d'après 1945 ne changea pas le profil de l'émigration serbe qui resta monarchiste et tchetnik. Le monarchisme et le "tchetnisme", devenus l'expression du nationalisme de la diaspora, constituaient un phénomène nouveau dans la vie politique des Serbes émigrés. Même la vie religieuse de l'émigration politique serbe était quelque chose de nouveau ${ }^{6}$.

Contrairement, par exemple, à l'émigration russe de 1917, qui était une émigration bourgeoise et aristocratique, l'émigration serbe, et, jusqu'à un certain point, l'émigration croate avaient un caractère populaire. Au point de vue politique, il est vrai que certains des représentants des partis existant dans le Royaume de Yougoslavie quittèrent le pays, mais leur activité politique fut réduite en émigration. Délaissant la discussion politique, les émigrés politiques serbes se regroupèrent autours de quelques principes fondamentaux: le nationalisme justifié par l'idée de la grandeur d'une Grande Serbie, l'attachement à la monarchie ou l'attachement à la démocratie.

\section{L'émigration croate}

15 L'émigration politique croate, tout au moins au départ, n'était pas aussi nombreuse. $\mathrm{Ne}$ serait-ce que du fait qu'une bonne partie des oustachis et de leurs familles, ainsi que les Croates domobranes ${ }^{7}$, qui s'étaient rendus aux forces britanniques à Bleiburg avaient été remis aux autorités communistes. Pratiquement personne ne survécut à la tragédie de Bleiburg, connue sous le nom de "marche vers la mort". Cependant, une grande partie des oustachis, la plupart des généraux et des ministres, avec à leur tête Ante Pavelić ${ }^{9}$, parvinrent à quitter la Croatie en suivant l'armée allemande. 

pourrait dire que l'émigration croate est la seule à avoir accompli une réconciliation nationale face à des conflits idéologiques. Autrement dit, elle a dépassé le conflit national qui avait eu lieu pendant la guerre civile (entre les communistes, les oustachis, les domobranes) grâce à la prééminence de l'idée d'un État croate indépendant. Les Croates ont adopté relativement tôt l'idée d'une "union des Croates" sans condition idéologique. Les premiers signes de ce désir d'un rapprochement croate dépassant les lignes idéologiques sont perceptibles dans les écrits de Mate Meštrović, particulièrement dans son livre La Croatie aujourd'hui et demain (1968), dans lequel il lança un appel pour mettre "fin à la division entre Croates communistes et nationalistes $»^{14}$. D'autre part, le consensus réalisé entre les communistes croates et les émigrés oustachis ${ }^{15}$ sur la création d'un État croate indépendant ${ }^{16}$ était fondamental pour que soit formé un Conseil national croate au sein duquel on parvint à une réconciliation de fait. Incontestablement, une particularité de l'émigration croate est d'avoir créé et fait fonctionner le Conseil national croate, organe directeur commun à l'ensemble de cette émigration, qui a opéré une réconciliation idéologique et nationale, alors que les divisions restent l'un des points les plus douloureux de toutes les émigrations de l'Europe de l'Est.

de l'émigration en fut simplifiée : le critère de regroupement était le fait d'être pour ou contre l'État indépendant de Croatie (NDH), État de taille variable selon les conceptions politiques.

n seul trait est commun aux émigrations politiques serbe et croate : jamais elles n'ont accepté le découpage national effectué par le régime communiste. Cela, naturellement, concerne en premier lieu l'émigration serbe la plus touchée par la création de nouvelles nations sur son territoire national, mais aussi les Croates qui n'acceptèrent jamais que les Musulmans soient une nation à part.

21 La composition sociale de l'émigration croate et serbe révèle que la plupart des émigrés de 1945 étaient des combattants attendant que survienne un conflit entre l'Est et l'Ouest, conflit qui serait dû à deux conceptions politiques antagonistes, la démocratie 
et le totalitarisme. Cette composante guerrière touchait plus l'émigration politique serbe que l'émigration croate. Et cela pour la simple raison que les Anglais livrèrent aux partisans de Tito plus de formations militaires croates, en majorité oustachies.

\title{
La genèse de l'émigration politique serbe et croate à travers l'analyse du système juridique et idéologique qui la produit
}

\author{
Un Parti communiste fort une fois arrivé au \\ pouvoir finit toujours, à plus ou mois brève \\ échéance, par liquider ses partenaires politiques \\ avant de s'emparer du pouvoir absolu qu'il ne \\ sera désormais plus question de partager avec \\ quiconque ni d'en discuter la légitimité. \\ Vladimir Boukovski
}

Le rapport entre la connaissance politique et la connaissance sociologique dans les pays

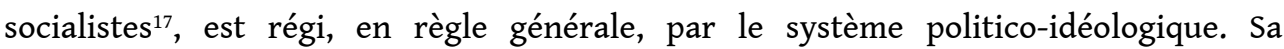
suprématie absolue fait de la connaissance sociologique, notamment en sociologie politique, une servante du système monopartiste. Par conséquent, leur rapport devient encore plus étroit quand le sujet d'étude est perçu comme la négation de l'idéologie régnante, ce qui fut, par exemple, le cas de l'émigration politique.

Bien entendu, pour pouvoir dépasser la pratique mentionnée, il faut construire une nouvelle approche historique, politique, juridique et sociologique. La science sociale dans les pays ex-socialistes doit se libérer de la propagande qui, à travers la socialisation politique, a implanté la haine dans les consciences de ses citoyens pour se protéger mentalement de tout ce qui a une connotation politique différente. Autrement dit, l'étude de l'émigration politique en provenance des pays d'Europe de l'et renvoie à l'étude du système qui produit cette émigration.

\section{Système juridique et idéologique yougoslave}

L'émigration politique yougoslave ne peut être appréhendée uniquement du point de vue des divergences d'opinions politiques manifestées par les émigrés par rapport au pouvoir établi en Yougoslavie, car les forces en présence ont un antagonisme beaucoup plus profond à la base de leur différenciation :

- premièrement, le pouvoir établi et les émigrés ont été des adversaires lors de la guerre civile, et les deux partis conservent un esprit combatif. Le pouvoir communiste vise ainsi à conserver les positions qu'il occupe et à justifier les purges qu'il a effectuées après la guerre. L'émigration, quant à elle, craint de perdre ce qui fonde idéologiquement son existence ;

- deuxièmement, le pouvoir établi et l'émigration sont des adversaires à la fois politiques et idéologiques. L'émigration politique est considérée comme une opposition ${ }^{18} \mathrm{du}$ seul fait qu'elle est contre le régime politique existant en Yougoslavie. Si on la tient pour telle, il faut souligner le fait que cette opposition ne s'est pas formée sur la base de partis politiques mais qu'elle se divise selon des lignes idéologiques. 
L'apparition de l'émigration politique est manifestement liée à la création de la seconde Yougoslavie. L'instauration d'un nouveau régime n'impliquait pas nécessairement une émigration politique. Mais, dès le départ, l'attitude adoptée par le Parti communiste au pouvoir sur les plans politique et idéologique à l'égard de ses anciens et futurs adversaires posa les bases de la création d'une émigration politique. Autrement dit, une étude portant sur l'émigration politique implique l'analyse du système politique qui en est à l'origine et de son fonctionnement. Nous ne nous attarderons ici que sur le phénomène de l'appareil répressif légalisé sur le plan juridique. À travers le droit, c'est la volonté politique et idéologique du parti dirigeant qui s'exprime.

\section{Le capital symbolique exécutif}

27 L'une des premières tâches du parti dirigeant a été de fonder en droit un partage inégal du pouvoir au sein de la société. Le capital symbolique, que possède chaque groupe politique et à travers lequel il affirme ses valeurs et ses buts idéologiques, ne suffit pas à assurer l'hégémonie complète du pouvoir. Il convient donc de créer un capital plus efficace encore grâce au droit, c'est ce que nous pourrions appeler le "capital symbolique exécutif". Celui-ci définit les conditions dans lesquelles doit s'exercer la contrainte subjective, quand les stipulations juridiques tendant à former et préserver l'hégémonie ne sont pas respectées ${ }^{19}$.

Une telle pratique a été rendue possible par ce que l'on appelle le droit révolutionnaire, né de la victoire du Parti communiste dans la guerre civile yougoslave, au nom de la protection du nouveau régime. Ce droit révolutionnaire permet la répression politique, moyen admis pour contraindre les citoyens à accepter le système. « Le pouvoir peut, en fait, sous prétexte de protéger la Révolution, (...) supprimer les fondements de la liberté intellectuelle et spirituelle et de cette façon réinstaller le vieux système fait de tromperies et d'opinions préconçues qui permet de contrôler les citoyens et donc de les déposséder de leur liberté. $»^{20}$

Il est contestable, d'un point de vue scientifique, d'assimiler régime monopartiste et totalitarisme ; toutefois le dogmatisme et la foi en leur propre infaillibilité des régimes monopartistes préparent le terrain au totalitarisme. L'unanimité absolue, qui s'est manifestée en Yougoslavie, dans la période d'après-guerre, à travers l'organisation du Front populaire n'était que le pendant de l'absence de liberté.

Aujourd'hui, peu de personnes savent avec certitude que la Yougoslavie est sortie de la guerre avec un certain pluripartisme et que celui-ci était autorisé par la loi. Mais il est encore moins connu que ce pluripartisme, ou plus exactement la possibilité de se grouper en différents partis politiques, n'a jamais été formellement supprimé, aucune disposition constitutionnelle ni aucune loi ne l'ont expressément interdit (...). En fait, le système pluripartiste et la libre activité des partis politiques d'opposition n'ont été reconnus publiquement comme légitimes qu'en tant que "pont provisoire" destiné à faire passer les masses du côté de l'avant-garde du prolétariat. ${ }^{21}$

Dans la Yougoslavie d'après-guerre, le système multipartiste se transforma en monopole d'un parti unique qui se servait des dispositions juridiques pour légaliser la répression idéologique.

31 En analysant les dispositions juridiques qu'adopta le Parti communiste pendant la guerre, à savoir les ordonnances de Foča en 1942, les documents de Krajina, le règlement de l'état-major général portant sur les tribunaux militaires en 1944, les 
résolutions créant un tribunal devant juger les crimes et délits commis à l'encontre de l'honneur national serbe, et enfin l'établissement par l'Assemblée nationale provisoire de la FNRJ (Federativna Narodna Republika Jugoslavije - République fédérative populaire de Yougoslavie) d'une loi sur les délits commis à l'encontre du peuple et de l'État, nous pouvons étudier le rapport qui existait entre le droit et la coercition, rapport qui prenait une tournure de plus en plus idéologique. Dans les ordonnances de Foča, apparaît pour la première fois le terme "ennemi du peuple" pour définir les citoyens yougoslaves qui, d'une manière ou d'une autre, aidaient l'ennemi, affaiblissaient le pouvoir et causaient du tort à la lutte de libération nationale. La protection de la Révolution et du nouveau régime fut assurée par la loi sur les crimes commis à l'encontre de l'État et du peuple, adoptée dès 1945, « sans toutefois que soient définis les délits qui seraient poursuivis et punis dans le cadre de ces dispositions $»^{22}$. La Constitution de 1946, largement inspirée par la Constitution soviétique, mettait l'accent sur l'usage qui doit être fait du droit pénal pour punir les «crimes contrerévolutionnaires " que sont les délits à caractère politique et économique ${ }^{23}$. D'après un représentant du groupe alors au pouvoir, « la législation se doit de protéger le pouvoir nouvellement mis en place en rendant impossible tout changement $»^{24}$. Le pouvoir des communistes, d'abord "pouvoir populaire" auto-proclamé, devint un pouvoir étatique et ceux qui pensaient autrement furent assimilés à des ennemis et à des traîtres.

Le nouveau régime, après la Seconde Guerre mondiale, en Yougoslavie, mais également dans les autres pays socialistes sous l'égide de l'URSS, mit l'accent sur l'instauration de son propre pouvoir dans tous les domaines, aussi bien sociaux qu'individuels. Cette prise de pouvoir déboucha sur l'établissement de la peur, méthode sûre de gouvernement, que ce soit sous la forme de la terreur ouverte ou d'une législation pénale perverse. Mais, «le plus fort ne l'est jamais suffisamment pour être le maître, s'il ne transforme pas sa force en droit, et l'observation des lois en une obligation ", disait Rousseau.

Il existe, à côté de l'appareil répressif d'État, légitime car reposant sur des dispositions "légales", un appareil idéologique d'État ${ }^{25}$. Celui-ci permet de propager les valeurs et les orientations que prône l'idéologie dominante. L'apport d'Althusser dans ce domaine est d'avoir englobé dans l'appareil idéologique des institutions spécifiques, telles l'école ou la famille, qui ne sont pas idéologiques en tant que telles mais qui peuvent contribuer à renforcer l'idéologie.

L'autre caractéristique importante d'un système monopartiste est son monolithisme, car le parti se confond avec l'État, la collectivité et la patrie, faisant ainsi apparaître, pour emprunter l'expression de Durkheim, des phénomènes pathologiques de trois types: l'interdiction de toute forme de pensée et de comportement autres, la dissidence, l'émigration politique.

\section{L'émigration politique et son idéologie}

Tandis que les communistes au pouvoir s'assuraient l'élimination d'une éventuelle concurrence de l'émigration politique grâce au système répressif, cette dernière posait ses règles idéologiques. Autrement dit, l'analyse de l'émigration politique et de son fonctionnement ne serait pas complète si on ne consacrait pas une attention particulière à son idéologie, car c'est l'idéologie qui, d'une part, cimente les groupes d'émigrés et, d'autre part, assure leur pouvoir et leur influence. 

conditionne l'approche méthodologique de sa vie politique. Puisque sa structure partisane ne reflète pas suffisamment sa structure politique, il est possible d'analyser l'émigration politique à travers son idéologie. "La pluralité des expressions permet la modalité et la diffusion de ce que nous pouvons appeler les idéologies "sociales" au détriment des systématisations proprement politiques. ${ }^{26}$ Nous adopterons l'hypothèse que toute idéologie tend à réaliser ses objectifs et à mettre en pratique ses valeurs dans le domaine politique. Dans ce cas, nous pouvons espérer trouver dans le champ politique les paramètres indiquant l'influence exercée par l'émigration politique. Un slogan politique conforme à une idéologie peut être une forme d'action dans la mesure où il symbolise des relations entretenues avec les alliés et des conflits existant avec les adversaires.

i) mener des actions pour mettre un frein à la domination de la classe dirigeante nouvellement formée en Yougoslavie ou pour la menacer. Nous pourrions appeler cette sphère d'activité de l'émigration politique les champs idéologiques qui sont de deux sortes: a) l'émigration politique a exprimé une idée politique particulière qui avait valeur de mouvement social, "novateur", l'anticommunisme ; b) la lutte idéologique des différents mouvements de l'émigration politique va de pair avec la lutte pour l'indépendance nationale ;

ii) mettre en avant ses propres positions et ses propres intérêts, ce qui, avec le temps, se ramène au système d'emprise idéologique existant au sein du groupe.

\section{L'émigration politique et ses champs idéologiques}

\section{L'anticommunisme}

L'anticommunisme, en tant que champ idéologique de l'émigration politique, s'appuie sur le nationalisme ou sur la démocratie. «Pour obtenir le soutien de l'opinion publique internationale, il ne suffit pas que l'émigration dise qu'elle est contre le régime communiste, il est indispensable qu'au même moment elle déclare au nom de quoi elle est contre ce régime et par quoi elle le remplacerait s'il venait à disparaître. $»^{27}$

\section{L'anticommunisme serbe}

Très souvent, il existe un lien étroit entre nationalisme et démocratie et le système social souhaité, comme c'est le cas chez les Serbes monarchistes. La majeure partie de l'émigration serbe est anticommuniste car le communisme est l'antithèse du monarchisme. Le refus du communisme n'est pas purement idéologique: il est empreint de nostalgie et de valeurs anciennes.

41 Le deuxième facteur qui caractérise l'anticommunisme chez les Serbes émigrés, comme chez une bonne partie de ceux qui n'ont pas quitté le pays, est l'origine nationale de Josip Broz. "L'origine ethnique, à elle seule, de Josip Broz explique son attitude automatiquement négative à l'égard du peuple serbe. (...) Cette conclusion constitue un a priori, elle est partiale et à sens unique. ${ }^{28}$ Précisément parce que cette conclusion est trop simple, s'est répandue la thèse d'un complot du Komintern et du Vatican, complot dirigé contre le peuple serbe auquel était imposé le communisme. 
Les groupes qui, au sein de l'émigration, parviennent à dépasser cette vision simpliste du communisme ne sont pas rares mais comptent peu de membres, et ont, donc, une influence restreinte au sein de l'émigration ${ }^{29}$. Il s'agit de groupes qui défendent des principes démocratiques, comme, par exemple, la ligue « Oslobođenje » (ce qui signifie "libération") ${ }^{30}$, le "Comité national yougoslave à Londres" ${ }^{11}$, et le groupe parisien des "Rencontres démocratiques" ${ }^{2}$. Il y a dans leurs engagements programmatiques la même opposition et la même réserve à l'égard du nationalisme ${ }^{33}$. Le "Zbor" (l'Union) de Ljotic ${ }^{34}$ entre dans ce cadre si ce n'est que, pour lui, l'opposition au communisme ne passe pas par la démocratie mais par l'autocratie d'un monarque et d'une classe.

En 1958, les émigrés politiques serbe, d'orientation yougoslave, fondèrent aux ÉtatsUnis (à Milwaukee) "le Comité yougoslave anticommuniste" qui adopta les principes programmatiques $\mathrm{du}$ "Comité national yougoslave de Londres". «La résistance du peuple au sein du pays et tous les efforts faits par les citoyens libres qui ont quitté la Yougoslavie doivent se concentrer davantage sur une lutte implacable contre le régime communiste et l'instauration de la liberté, de la justice sociale et de la démocratie en Yougoslavie (...). Il faut mener le combat contre le communisme au nom des principes de la démocratie contemporaine. $»^{35}$

\section{L'anticommunisme croate}

Les Croates étaient anticommunistes parce que leur postulat idéologique assimilait la Yougoslavie aux Serbes, donc, si la Yougoslavie était communiste, les Serbes étaient les porteurs du communisme yougoslave.

À partir de 1969, du fait des événements en Yougoslavie, l'émigration politique croate arrive à un tournant très intéressant. Elle conserve un anticommunisme dirigé contre la "Serbo-slavie", mais pas contre les communistes croates. Un compromis s'instaure sur la base de l'unité nationale, le fait de réclamer la souveraineté croate sert à mesurer le patriotisme. Le lien entre communisme et nationalisme n'était, d'ailleurs, pas un phénomène nouveau : il avait été particulièrement fort dans la période de l'entre-deuxguerres quand l'objectif de base des communistes yougoslaves, à partir du congrès de Dresde en 1926, avait été de détruire la Yougoslavie en accordant la souveraineté aux petits peuples. Ce même principe de reconnaissance des petits peuples au détriment des grands a été adopté dans la Yougoslavie d'après-guerre ${ }^{36}$.

En mettant l'accent sur la coopération entre les communistes croates et les oustachis pour démanteler "l'État yougoslave artificiel", dans laquelle le national prédominait sur l'idéologique, on souligne que: « en ce qui concerne l'objectif national de l'État croate souverain, les oustachis et les communistes, s'ils pensent de façon nationaliste, diffèrent peu $»^{37}$.

Pour mettre en oeuvre l'idée d'une réconciliation nationale, on compte, avant tout, sur la jeune génération :

Ces nouveaux Croates, les fils des anciens oustachis, des anciens domobranes et des anciens partisans, nourrissent, dans leur coeur pur, un amour pour la Croatie, et, grâce à leurs jeunes forces, ils franchiront l'abîme qu'ont creusé leurs grands-pères et leurs pères, abîme que, des deux côtés, des étrangers ont approfondi et qui a, malheureusement, séparé les membres d'un même peuple (...). Car, aujourd'hui, les uns et les autres comprennent qu'en 1945 ce ne sont pas les oustachis qui ont été écrasés, ni les communistes qui ont vaincu, mais, en réalité, c'est bien la Croatie qui a été défaite. ${ }^{38}$ 


\section{Le nationalisme} nationale et l'anti-yougoslavisme.

\section{L'indépendance nationale}

49 l'émigration politique est liée à un nationalisme qui est une forme particulière d'idéologie. Le nationalisme est au centre du champ idéologique de l'émigration politique. "Les sentiments nationaux sont vécus souvent non comme des vérités subjectives, disons comme une conviction intime, (...) mais, au contraire, comme des vérités objectives. $»^{39}$

50 Les idéologies nationalistes combattent directement les troubles liés à l'incertitude du moi dans les situations désespérées. Elles communiquent à chacun une identité rattachée à des idéaux, qui permet d'accepter des sacrifices et même la mort. La destruction devient une valeur suprême, comme on l'a vu chez les terroristes, et cela au nom des idées nationalistes. Les nationalismes serbe et croate présentent la tragédie vécue par leur peuple "d'une manière empreinte d'auto-apitoiement, d'un sentiment de revanche et d'orgueil national poussé à l'extrême $»^{40}$.

51 «La souveraineté et le nationalisme sont les vecteurs fondamentaux du phénomène actuel des réfugiés. $»^{41}$ Le nationalisme chez les Croates (dans le sens de la création d'un État national) et chez les Serbes (dans le sens de la préservation d'un État unitaire) est grevé du poids de l'idée d'un État souverain. Les deux peuples font référence au modèle français d'instauration d'un État-nation (mot que les nationalistes emploient dans le sens d'État national ${ }^{42}$ ) sans se rendre compte que ce n'est qu'un des principes posés par la révolution française. Le deuxième principe, tout aussi important, que sont les droits de l'homme, est totalement rejeté par les Serbes au nom de l'État et par les Croates au nom de la nation qui doit déboucher sur la création d'un État. En réalité, ces deux tendances cachent une vision jacobine de l'État, elles subordonnent tout à un Étatincarnation de la volonté nationale proclamée. La terminologie idéologique, dans ce domaine, regorge de glorifications pseudo-romantiques du passé et déborde d'un sentiment de supériorité morale. Tout sert le culte de l'État national.

\section{L'anti-yougoslavisme}

La deuxième yougoslavisme.

Les Serbes “d'acier" 43 ont une attitude doublement négative à l'égard de l'idée yougoslave. Premièrement, ils assimilent gauchisme et État yougoslave, comme le fait le leader des Serbes des États-Unis, S. Draškovićn ${ }^{44}$ : «l'idée yougoslave [est] une arme pour démembrer le peuple serbe. C'est pourquoi ceux qui la recommandent aux Serbes (...) sont, en général, les mêmes qui étaient et sont encore aujourd'hui des partisans du régime communiste en Yougoslavie ${ }^{45}$. Le deuxième postulat serbe anti-État yougoslave repose sur les conséquences tragiques de l'attitude de l'État Indépendant de Croatie envers les Serbes; ce qui a débouché sur le slogan «plus jamais avec les Croates $! »^{46}$. Ainsi une méfiance extrême envers les Croates s'instaure et les Serbes qui 
vivent en dehors de la Serbie, au-delà de la Drina, allient toute dissolution éventuelle de la Yougoslavie avec la création sans condition d'une Grande Serbie qui serait leur protectrice ${ }^{47}$. Une bonne partie de l'émigration serbe extrémiste ne souhaite pas faire la différence entre Croates et oustachis, ce qui approfondit encore le gouffre entre ces nationalités. en serbe, en faisant de lui la source de toutes les difficultés que connaissent les Croates. L'anti-serbisme chez les Croates, outre des raisons historiques $^{49}$, s'appuie sur le fait que les Serbes sont le peuple le plus nombreux en Yougoslavie pour les présenter comme la source et le vecteur de l'inégalité nationale. Il est intéressant de noter que chez les peuples numériquement plus petits (les Slovènes ou les Macédoniens), la résistance à l'hégémonisme serbe n'est pas aussi manifeste que chez les Croates. Naturellement, cela témoigne de la forte identité nationale du peuple croate qui pourrait, éventuellement, devenir prépondérant en Yougoslavie ou dans les Balkans si les Serbes n'avaient une identité nationale plus forte encore. B. Radić l'a fort bien exprimé : « le milieu dont je suis issu aimait les Serbes et, par conséquent, je les ai aimés, mais aujourd'hui je suis anti-serbe. Pourquoi ? Parce que tout Croate en contact avec des Serbes se sent appartenir à un peuple de troisième zone $»^{50}$.

Une des composantes du nationalisme croate est l'oppression économique dont est, selon ses représentants, victime la Croatie. Ils ont ainsi crée une psychose selon laquelle les Croates ont, en Yougoslavie, une position inférieure à celles des Serbes : « à l'instar de tous les peuples asservis, les Croates sont contraints de soutenir et d'entretenir la police, l'armée et les fonctionnaires serbes, leur désolante absence de liberté $»^{51}$.

\section{Le système d'emprise idéologique au sein des groupes émigrés}

Le besoin, propre à l'émigration, de mener une large activité idéologique ne répond pas seulement à la nécessité de justifier la situation de fait dans laquelle s'est trouvée l'émigration. D'ailleurs, si cela avait été le seul contenu de cette idéologie, elle aurait vite été assimilée à de la démagogie. L'émigration a besoin d'objectifs et de valeurs correspondant à une vision presque ontologique de l'avenir. «La position sociale des acteurs sociaux entraîne d'abord l'apparition de ce qu'on peut appeler des effets de perspective. $\|^{52}$ Le fait de se tourner vers l'avenir qui, d'ailleurs, pour nombre de groupes émigrés, n'est qu'un retour au passé, permet une transmission idéologique où l'idolâtrie de l'avenir ne fait que compenser une réalité défavorable. L'idéologie peut mobiliser ses partisans autour d'une action sociale et les pousser à l'abnégation parce 
qu'elle n'est pas seulement un système d'idées rationnellement organisées, mais possède également une dimension affective dont découle son pouvoir. L'idéologie permet une identification personnelle plus facile en particulier dans les moments de crise. Apparaissent des symboles-clés, des slogans, on en appelle à des mythes et des légendes, ainsi qu'au messianisme de sa nation, le tout permettant de reconnaître à leur vocabulaire idéologique ceux qui pensent de la même façon. Tout est fait pour donner une image négative des adversaires et souligner sa propre valeur.

La deuxième caractéristique importante de l'idéologie de l'émigration est qu'elle présente la société comme étant l'objet d'une confrontation idéologico-politique aiguë, ce qui complète le vécu affectif de l'émigré. Cela permet d'effacer dans la conscience des partisans de cette idéologie la différence entre ennemi idéologique et adversaire personnel. Ce processus est totalement identique à celui qui avait lieu en Yougoslavie, où l'image de la réalité sociale était divisée de façon manichéenne en "nous" (communistes) et "eux" (les émigrés politiques). "La violence symbolique dirigée contre les ennemis confirme l'unité du groupe et sert à sa cohésion comme les appels persuasifs à mobiliser les énergies contre les opposants et les indifférents. ${ }^{53}$ Le groupe est présenté comme le détenteur de valeurs intangibles et dès lors comme le détenteur du droit à la vérité.

D'autre part, "par son aspiration effrénée à la vérité absolue, l'idéologie "corrige" la réalité tout entière, et cette image de la réalité (...) devient indépendante : elle se libère de l'objet et le remplace $\aleph^{54}$. Cependant, la réalité se charge elle-même de détruire les vues "corrigées" qu'on a d'elle, de sorte qu'il faut toujours ajouter à l'idéologie de nouveaux postulats pour expliquer pourquoi les buts proclamés ne sont pas atteints. Avec le temps, ces objectifs "glissent" et affaiblissent la cohérence politique de nombreux groupes d'émigrés qui deviennent des rassemblements folkloriques ${ }^{55}$ conservant en mémoire la raison pour laquelle leurs membres ont émigré et des souvenirs particulièrement forts de la période de la guerre.

61 L'impact de l'idéologie dépend de nombreux facteurs dont le plus décisif est la capacité qu'a le groupe à intégrer ses membres. Plus un groupe atteint un haut niveau de cohésion $^{56}$, plus il est isolé des autres groupes (ce qui est le cas en Amérique et en Australie) et plus l'impact de l'idéologie est fort. «L'idéologie, quelle qu'elle soit, nous installe dans un univers moralement sûr et intellectuellement confortable où nos comportements sont justifiés et nos désirs légitimés. ${ }^{57}$ Pour atteindre l'interdépendance et l'intégration la plus grande entre leurs membres, les groupes d'émigrés, en plus du symbole qui les différencie des autres groupes ou des autres nations, fonctionnent suivant le système éprouvé de l'exclusivisme, et pour souligner leur propre valeur, dénigrent les autres.

\title{
L'utilisation des émigrés de l'Est dans la guerre froide
}

\author{
Si, dans la pratique, la manipulation des hommes \\ et des organisations restait essentiellement une \\ tâche des services secrets, le traitement du \\ problème en général leur échappait pour passer \\ au niveau du pouvoir politique.
}


62 Au début des années 1950, une politique d'aide aux émigrés politiques, dits anticommunistes, en vue de leur utilisation dans la guerre froide et du renforcement systématique de leurs organisations fut appliquée par chaque pays occidental, y compris le Vatican. En quelques années seulement, on créa nombre d'associations, de comités, de fonds et de fondations pour que l'émigration puisse être utilisée, soit idéologiquement, soit de façon subversive contre le bloc communiste ${ }^{58}$. Cette politique ayant échouée, il fallut montrer plus de vigilance envers le système idéologique qu'était le système communiste.

\section{Les différentes organisations pour l'utilisation des émigrés}

La stratégie d'utilisation de l'émigration politique pendant la "guerre froide" avait pour but de renforcer l'opposition dans les pays socialistes et donc de disposer d'un facteur de déstabilisation idéologique de l'adversaire.

64 Le problème de l'utilisation politique des réfugiés fut posé durant la Conférence de Londres d'avril - mai 1951. Lors de la quatrième réunion, les ministres des Affaires étrangères prirent, à ce sujet, une décision de principe : «bien que les émigrés et les réfugiés politiques des pays satellites doivent être utilisés avec précaution, la possibilité existe d'en faire un usage judicieux. Le Royaume Uni, les États-Unis et la France devraient coordonner leurs attitudes sur ce point dans le but de faire l'usage le plus complet des émigrés dans l'intérêt de la politique occidentale $»^{59}$.

L'intérêt de la politique occidentale était d'utiliser à tout prix l'émigration politique dans la lutte contre l'Est. C'est la raison pour laquelle ils donnèrent à tous les exilés un même statut. Résistants et collaborateurs ${ }^{60}$ furent mis sur le même pied. D'autre part, il faut ajouter que parmi les animateurs de l'émigration politique anti-communiste, les extrémistes et les gens ayant sympathisé avec l'hitlérisme étaient plus nombreux que les libéraux.

Le 7 mars 1951, le New York Times annonça la création du «Comité américain pour la liberté des peuples de l'URSS » dont un des présidents était l'ancien secrétaire d'État adjoint Howland Sargeant. Lors de son entrée en fonction, il déclara: «le comité étendra ses activités et essaiera dans tous les domaines de coopérer avec les émigrés anti-communistes venus d'Union soviétique. Il le fera en appliquant la politique américaine d'autodétermination des peuples de l'URSS ».

\section{Le droit des peuples à disposer d'eux-mêmes}

67 Le droit des peuples à disposer d'eux-mêmes a toujours été lié à la démocratisation, à la libération et à la démocratie, mais, dans la guerre froide, ce droit proclamé acquit une force idéologique et devint un enjeu politique. Les vrais problèmes posés par l'autodétermination politique ont donc aujourd'hui toute chance de concerner des situations postérieures à la "libéralisation" des régimes communistes et même des pays non communistes. Il ne faut pas oublier que les États successeurs des anciennes puissances communistes sont des États multinationaux du point du vue ethno-culturel et linguistique dont la composition ethnique et les frontières politiques furent déterminées par décision du Parti communiste et des autorités. Les références aux Droits de l'Homme et au droit des peuples à disposer d'eux-mêmes furent une arme dont usèrent de façon privilégiée les États dits capitalistes pour combattre le 
socialisme, surtout après la signature de l'acte final d'Helsinki. Dans l'état actuel, le droit à l'autodétermination des peuples s'identifie au nationalisme, à un but purement idéologique. Pratiquement dans tous ses écrits, l'émigration politique fait référence au droit découlant de l'autodétermination ce qui, dans ce cas, signifie l'émancipation nationale par rapport à un État. Les Droits de l'Homme et ceux des peuples sont opposés à l'État, surtout selon une conception hobbesienne de la souveraineté de l'État communiste. L'État communiste est considéré comme un obstacle à l'exercice de ces droits.

L'utilisation des émigrés politiques s'est faite à deux échelons différents : idéologique, c'est-à-dire la possibilité politique de les faire participer au pouvoir dans leur pays d'origine ; militaire.

L'utilisation militaire des émigrés politiques jouit d'une assez grande attention de la part des pays occidentaux. Cette question pouvait trouver trois solutions : i) l'utilisation des émigrés politiques au sein de l'OTAN, parce que "certaines organisations d'émigrés provenant de démocraties populaires ont fait à plusieurs reprises des offres de service au SHAPE $»^{61}$; ii) chaque pays qui avait admis sur son territoire des réfugiés devait se préoccuper du traitement qu'il conviendrait de leur réserver en temps de guerre; iii) la création d'une "Armée européenne de Libération"62. Les Américains voulurent faire stationner ces troupes comprenant six divisions d'abord en Allemagne, puis en France. Mais, en raison de la nouvelle politique étrangère de l'URSS, ce projet fut mis en sommeil ${ }^{63}$.

Néanmoins, chacun de ces projets souleva certaines difficultés. D'abord, la totalité des pays signataires de l'OTAN entretenaient des relations officielles avec les démocraties populaires. Les émigrés politiques ne cachaient par leur hostilité à l'égard des régimes instaurés à l'Est, mais le Pacte de l'Atlantique Nord avait officiellement un caractère défensif. La constitution d'unités d'émigrés politiques au sein de l'OTAN favorisait une hostilité ouverte à l'égard de l'URSS.

71 Il semble qu'il aurait été politiquement plus opportun d'aborder ce problème au niveau national, c'est-à-dire celui des États qui avaient accueilli des réfugiés. L'essentiel était de le faire discrètement.

\section{Le cas yougoslave}

72 Le 10 octobre 1951, à Washington, le Président des États-Unis, Harry Truman, signait une loi dite de "Sécurité mutuelle". Le fameux article 101 de cette loi prévoyait que l'on pouvait prélever sur le budget de la "Sécurité mutuelle » ${ }^{64}$ une somme de cent millions de dollars. Cette somme devait être distribuée au profit de personnes résidentes ou évadées d'URSS, de Pologne, de Tchécoslovaquie, de Hongrie, de Roumanie, d'Albanie, de Bulgarie et des pays Baltes ou des zones d'Allemagne et d'Autriche sous occupation communiste. La Yougoslavie n'était pas incluse dans ce programme.

73 C'était le même cas avec l'ACEN (Assemblée des nations captives d'Europe) ou avec Radio Free Europe, avec l'organisation "Nations captives", ou avec le "Bloc antibolchévique des nations". Les émigrés yougoslaves n'avaient pas accès aux organisations des émigrés des pays de l'est. La question yougoslave était traitée de la même façon au Foreign Office à Londres qu'au Carnegie Hall à New York ou au Quai d'Orsay à Paris. Dans l'esprit des dirigeants occidentaux, le régime de Tito devait 
basculer dans le camp occidental et il ne fallait pas encourager l'émigration politique yougoslave opposée à Tito.

74 Pour démontrer l'importance de la Yougoslavie de Tito, qui s'était détachée de la zone d'influence de l'Union soviétique, la presse américaine dans sa fameuse "Déclaration de Libération", signée par les émigrés yougoslaves, nous présente les faits ainsi : «Il est à noter que les réfugiés yougoslaves ont également signé cette déclaration et plusieurs journaux américains ont relevé ce fait pour souligner que la Yougoslavie n'était pas un satellite mais au contraire un ennemi mortel de l'URSS ${ }^{65}$.

À cette époque, Tito obtint le soutien politique et économique des pays occidentaux, notamment des États-Unis ${ }^{66}$. Beaucoup de journaux de l'émigration dénonçaient «la naïveté et les illusions " des Occidentaux et, même, la «complaisance coupable » des responsables politiques qui permettaient à Tito de rester au pouvoir. L'émigration yougoslave se sentait isolée. Il fallait qu'elle trouve seule ses propres moyens de combattre le communisme et en particulier le titisme. «L'activité de l'émigration ne peut compter sur l'encouragement hypothétique de Free Europe. Nous sommes donc acculés à agir sans le soutien et malgré la politique de l'Occident entrée en usage à l'égard de la Yougoslavie. C'est pourquoi nous n'avons pas le droit de rêver d'une quelconque croisade contre le communisme. ${ }^{\prime 67}$

76 En tous cas, si l'utilisation des émigrés venus d'au-delà du "rideau de fer" n'est pas à négliger, l'émigration de l'est restait néanmoins divisée par la question politique. C'était le cas des émigrations polonaise, hongroise, roumaine et bulgare. Quant à la division de l'émigration yougoslave, elle était plus complexe.

Les divergences et désunions au sein de l'émigration politique permettaient à chaque groupe d'entreprendre ses propres actions en vue de l'indépendance de son pays. A posteriori, les Occidentaux continuaient de faire très peu de cas des milieux de l'émigration " estimant qu'au cas où des bouleversements surviendraient ils auraient très peu de chance de participer à l'exercice du pouvoir ${ }^{68}$.Pour les émigrés politiques, il ne restait rien d'autre à faire que d'accepter la réalité ou attendre un nouveau bouleversement mondial.

\section{Conclusion}

Ce bouleversement mondial, tant attendu, est venu à la fin des années 1980. Quelle était la capacité de l'émigration politique à y répondre?

D'abord, l'absence de guerre pendant 40 ans avait affaibli l'émigration politique issue majoritairement d'anciens groupes militaires. Ensuite, l'émigration politique avait nécessairement pris d'autres formes de lutte en se déclarant obligatoirement "démocratique". Sous ce couvert, elle a essayé d'introduire ses idées politiques ainsi que d'apporter son soutien économique aux nouveaux gouvernements. Le succès de cette alliance dépendra des relations établies pendant la période communiste ainsi que de l'adéquation du capital idéologique entre les nouveaux régimes et les émigrés.

80 Avec l'introduction du système multipartiste, l'émigration politique disparait de la scène politique car le débat politique est transféré dans le pays. L'émigration politique perd sa caractéristique première qui était d'être une opposition au système communiste, opposition basée essentiellement sur les problèmes de la question nationale et de la démocratie. Cet ensemble d'hypothèses, cependant, ne se vérifie pas 
dans le cas serbe. En effet, bien qu'en Serbie ait été introduit un système multipartiste, l'émigration politique reste hostile à S. Milošević et à son système "communiste" et se voit toujours comme une opposition. "Il y a, chez les Croates, plus d'émules des oustachis que, chez les Serbes, de partisans des tchetniks. Parmi les Serbes émigrés, il y a, aujourd'hui, peu de partisans deMilošević, la plupart d'entre eux ont une vision raisonnable et démocratique de la situation actuelle en Yougoslavie. $»^{69}$

Bien qu'elle soit issue du mouvement oustachi, ce qui lui donne une connotation négative, l'émigration croate a atteint, à la différence de toutes les autres émigrations d'Europe de l'est, un niveau enviable d'organisation et d'adaptabilité. Son apport à la question nationale croate est multiforme et elle remplit toutes les conditions d'une émigration politique réussie. La création du Conseil national croate (HNV - Hrvatsko Narodno Vijeće), qui a opéré un tournant déterminant en réalisant une réconciliation nationale, a ouvert la route au retour des émigrés politiques croates dans leur pays. L'émigration politique croate a eu sa chance historique et elle ne l'a pas laissée passer, à la différence de l'émigration serbe qui est restée anticommuniste alors même que le communisme est en train de se transformer de façon décisive.

82 L'hégémonisme serbe qui, dans le Royaume de Yougoslavie de 1918 à 1941, se manifestait par la prépondérance des Serbes sur les plans politique et militaire, se fait également sentir dans l'émigration. Les Serbes se désintéressent tout simplement de certaines questions qui, s'ils n'étaient pas sûrs de leur suprématie, seraient des questions politiques des plus normales. Les Serbes comptent: sur leur supériorité numérique par rapport aux autres peuples de Yougoslavie (ils constituaient $43 \%$ de la population de ce pays), et sur leur passé de vainqueurs qui jouissent de la sympathie des Alliés de la Première et de la Deuxième Guerres mondiales. L'apolitisme relatif, malgré son anticommunisme, de l'émigration serbe débouche sur la création de mythes, la pensée politique se meut plus sur un plan utopique qu'idéologique. Les Serbes continuent, dans l'émigration, à se battre entre eux autour des questions de la couronne, du partage du pouvoir et de l'organisation de l'État. Les Serbes n'ont pas réussi, ni à l'intérieur, ni à l'extérieur du pays, en émigration, à dépasser une sorte de régionalisme (ils se subdivisent suivant leur région d'origine, il y a les Serbes de Serbie, originaires de Šumadija, de Voïvodine, de Kosovo, plus les Valaques, ..., et les Serbes hors de Serbie, originaires de Lika, de Kordun, de Banija, de Bosnie, d'Herzégovine, du Monténégro, de Macédoine, ...), de sorte qu'il s'agit d'un peuple "inachevé" car il n'a pas d'intérêt national global.

L'idée d'une Grande Serbie ne constitue pas un programme national comme on le pense généralement. Les Serbes n'ont jamais élaboré de programme national complet. Même dans le "plan" de Garašanin, "Dessein" (Načertanije), qui, d'ailleurs, expose plus une certaine conception d'un État à construire qu'un programme national, le combat des Serbes pour l'idée yougoslave et l'unification de tous les Serbes n'est pas, de toute évidence, dépourvu d'une certaine dualité. 


\section{NOTES}

1. L'étude complète de cette question est faite dans mon livre (Glamočak (Marina), Koncepcije Velike Hrvatske $i$ Velike Srbije u političkoj emigraciji (Les conceptions de la Grande Croatie et de la Grande Serbie dans l'émigration politique), Užice : KIC, 1997.

2. Karapandžić (Bor), Kočevje, Titov najkrvaviji zločin (Kočevje, le plus grand crime de guerre de Tito), Cleveland, 1959.

3. Knežević (Radoje), «Podaci o jugoslovenskoj emigraciji » (Données sur l'émigration yougoslave), Poruka, (52), décembre 1958, p.13.

4. Dragoljub Mihailović, alias Draža (1893-1946), colonel de l'Armée royale yougoslave, fut le chef de la résistance serbe (1941-1945) avant d'être nommé général (1941) puis ministre de l'Armée et de la Marine (1942) par le gouvernement royal yougoslave en exil et de devenir le commandant en chef de l'Armée yougoslave dans la patrie, majoritairement composée de Serbes. Au lendemain de la guerre, accusé d'être un criminel de guerre, il fut arrêté et emprisonné par les communistes. Condamné à mort, il fut exécuté le 17 juillet 1946.

5. Conversation avec Desimir Tosić, in Galić (Mirko), Politika u emigraciji (La politique au sein de l'émigration), Zagreb: Globus, 1990, p.102 (D. Tosi\}, rédacteur de la revue Naša reč, est le seul émigré politique serbe qui soit rentré en Serbie et y ait fait de la politique. Il est devenu viceprésident du Parti démocrate et député à l'Assemblée fédérale).

6. Cf. le chapitre sur la scission au sein de l'Église orthodoxe serbe in Glamočak (Marina), op cit.

7. Pendant la Seconde Guerre mondiale, les Domobranes étaient une milice restée fidèle aux idées du Parti paysan croate.

8. Voir Nikolić (Vinko), « Blajburska tragedija hrvatskog naroda » (La tragédie du peuple croate à Bleiburg), Hrvatska revija, 1976; Nikolić (Vinko), Bleiburg, les causes et les conséquences, Barcelona / München : Knji ica Hrvatske revije, 1988.

9. Ante Pavelić (1889- 1959), membre du Parti croate du droit, avait repris l'idéologie d'Ante Starčević. En 1927, il fut élu député au Parlement central de Belgrade. En 1929, il s'exila. Sous l'égide de Mussolini et du régent hongrois Horthy, il créa l'organisation terroriste oustachie responsable de l'assassinat du roi Alexandre I ${ }^{\text {er }}$, à Marseille, en 1934. En 1941, aidé par les Italiens, puis les Allemands, il devint le Poglavnik (ce qui signifie Führer, Duce) d'une Croatie satellite de l'Axe. Il y mena une extermination systématique des Serbes, des juifs et des Tsiganes. Il réussit à s'enfuir de Yougoslavie en 1945 et se cacha dans de nombreux pays avant de mourir à Madrid.

10. Voir Paris (Edmond), Le Vatican contre l'Europe, Paris: Librairie Fischbacher, 1969, pp. 317-341; Clissold (Stephen), Croat Separatism: Nationalism, Dissidence and Terrorism, London: Conflict Studies, (103), january 1979.

11. Nikolić (Vinko), «Za državnu samostalnost i slobodu Hrvatske » (Pour la souveraineté étatique et l'indépendance de la Croatie), Hrvatska revija, 1, mars 1952, p. 11.

12. Clissold (Stephen), op.cit. ; SSIP, PA confidentiel, 1957, F-II.

13. Le Conseil national croate a été fondé le 3 février 1974. Son premier président était Ante Dosen, un proche collaborateur de Pavelić. Le Conseil national croate prétendait surmonter les rivalités existant dans l'émigration. L'organisation est devenue un corps commun à la direction duquel participaient des membres de l'ex-régime oustachi, des membres de l'émigration issue du printemps de 1971, ainsi que les membres des organisations terroristes.

14. Stefanović (D.), Les origines de la crise croate de 1971, thèse de doctorat, Paris, 1979, p. 358.

15. Certaines recherches montrent que les communistes et les oustachis avaient commencé à collaborer sous la dictature du Roi Alexandre quand les deux partis avaient été interdits. Cette collaboration se poursuivit pendant la Seconde Guerre mondiale. Voir Lazić (Branko), Titov 
pokret i rě̌im u Jugoslaviji (Le mouvement titiste et le régime en Yougoslavie de 1941 à 1946), Paris : s.e., 1946.

16. Le Programme de l'opposition démocratique croate, paru en 1968 sous la forme d'une brochure, affirmait qu'une collaboration s'était engagée entre les forces d'opposition existant à l'extérieur et celles existant à l'intérieur de la Croatie.

17. La notion de connaissance politique désigne l'expression de la pensée dominante sacralisée dans les systèmes à parti unique. Les connaissances en sciences sociales dans lesquelles s'est infiltrée la politique sont devenues un instrument politique qui ne permet pas de porter un regard critique sur l'idéologie dominante.

18. Le concept d'opposition, dans le contexte de l'émigration politique et du mouvement dissident, a une signification plus large que celle que lui donne la théorie sociologique usitée en Occident et qui le définit dans le cadre d'un système parlementaire. La pluralité des expressions que l'on trouve au sein de l'émigration politique permet la mobilité et la diffusion de ce que nous pouvons appeler des idéologies sociales au détriment de systématisations proprement politiques.

19. Govedarica (Selimir), "Coercition et non-coercition », texte non-publié, CNRS, p. 11.

20. Tadić (Ljubomir), Tradicija i revolucija (Tradition et révolution), Beograd: Srpska književna zadruga, 1972, p. 199.

21. Koštunica (Vojislav), Čavoški (Kosta), Stranački pluralizam ili monizam (Pluripartisme ou monopartisme), Ljubljana : Tribuna, s.d. (fin 1980).

22. Ibid., p. 38.

23. Bavcon (Ljubo), ur., Kaznjenopravna zaštita države i njenog državnog uređenja (La protection de l'État et du régime dans le droit pénal), Zagreb : Globus, 1987, p. 202.

24. Ibid., p. 38.

25. Althusser (Louis), Les appareils idéologiques d'État, Paris : La pensée, 1970, p. 12.

26. Ansart (Pierre), Les idéologies politiques, Paris : PUF, 1974, p. 97.

27. Poruka, (50-51), 1958, p. 12.

28. Inić (S.), «Tito i Srbi » (Tito et les Serbes), in Đilas (Aleksa), ur.,Srpsko pitanje (La question serbe), Beograd : Politika, 1991, p. 198.

29. Les groupes qui, dans l'émigration, se battent contre l'extrémisme, qu'il soit nationaliste ou communiste, sont qualifiés par le régime communiste de "centres de guerre psychologique". Voir Mičović (V.), « Protiv svoje zemlje » (Contre son pays), Politika ekspres, 16/01/86, p. 16.

30. En 1947, à Paris, fut fondée l'association serbe Oslobođenje qui, du $1^{\text {er }}$ janvier 1948 jusqu'en 1990, a édité la revue Naša reč. En 1959, l'association prit le nom de Ligue Oslobođenje. En 1958, la Ligue ainsi que la revue s'installèrent à Londres. Oslobođenje a aussi créé une collection dite Bibliothèque Naše delo. En 1990, Oslobođenje a appelé ses membres à adhérer au Parti démocrate en Yougoslavie.

31. Jugoslovenski narodni odbor (JNO) - Comité national yougoslave, fondé à Londres par des membres de l'ancien gouvernement du Royaume de Yougoslavie, dirigé par Slobodan Jovanović. L'organe de cette organisation est la revue Poruka ("Le Message"). Le JNO se considère comme le représentant légal $\mathrm{du}$ "peuple prisonnier dans le pays" bien qu'aucun représentant des émigrations politiques croate et slovène n'y ait adhéré, à l'exception de quelques personnalités favorables à l'idée yougoslave. Voir « Les principes du Comité national yougoslave », Poruka, avril 1950.

32. Le groupe des Rencontres démocratiques est une aile dissidente d'Oslobođenje qui s'est créé quand ce dernier s'est installé à Londres. Les membres des "Rencontres démocratiques" collaborent avec le "Groupe de Paris". Celui-ci, composé de Bulgares, d'Estoniens, de Hongrois, de Lettons, de Lituaniens, de Polonais, de Russes, de Tchécoslovaques et de Yougoslaves, s'était constitué dès 1972 pour suivre les négociations de la Conférence pour la Sécurité et la Coopération en Europe. Voir La Conférence internationale des militants politiques de l'Europe du Centre et de l'Est, Strasbourg, 3-5 octobre 1975, p. 1. 
33. «Notre devoir national est de travailler obstinément à se dégager de l'obscurantisme, des préjugés nationalistes éculés, de l'esprit de clocher, de tout ce qui nous a été imposé de l'extérieur, ainsi que de notre propre primitivisme, de notre société et de nos régimes autoritaires. », « 20 godina stava i rada Saveza "Oslobođenje” » (20 ans de prises de position et de travail de l’Union “Oslobođenje”), Naša Reč, 1970, p. 19.

34. Dimitrije Ljotić s'est inspiré des idées de Charles Maurras. Les partisans de Ljotić sont des monarchistes et des yougoslaves intégraux. Pendant la guerre, ils ont collaboré avec les Allemands. Pour plus de détails, voir Glamočak (Marina), "A View on the Serbian Political Emigres ", in Grečić (Vladan), ur., Seobe Srba nekad i sad (Migrations serbes hier et aujourd'hui), Beograd : Institut za međunarodnu politiku i privrednu / Matica iseljenika Srbije, 1990.

35. Poruka, (47), 1958, p. 7.

36. Voir sur ce problème Lazić (Branko), op.cit. et Trivunac (Stevan), Serbian People today, Melbourne : Srpska misao, 1973.

37. Bušić (Bruno), Rien que la Croatie, Toronto / Zürich / Rome / Chicago : ZIRAL, 1983, p. 606.

38. "Requiem svim palima za Hrvatsku ", (Requiem pour ceux qui sont tombés au nom de la Croatie), Hrvatska revija, (1), 1970, p. 6.

39. Boudon (Raymond), L'idéologie ou l'origine des idées reçues, Paris : Seuil, 1992, p. 213.

40. Đilas (Aleksa), ur.,op.cit, p. 177.

41. Gerald (Dicks E.), Canada's Refugee Policy - Indifference or Opportunism ?, Montréal / London : Mc Gill - Queen's University Press, 1977, p. 2.

42. Apparemment il y a confusion entre les notions d'État-nation et d'État-national.L'État-nation peut être national ou multinational, il est toujours l'État d'une seule nation qui cherche à réaliser, par delà l'unité formelle, une unité nationale à la fois subjective et objective. L'État-nation désigne l'État moderne présentant certaines caractéristiques, notamment celle de correspondre à une nation et de se fonder sur le principe de la souveraineté nationale ou populaire.

Par contre l'État national est défini d'abord juridiquement - c'est l'État dont la Constitution ne proclame pas l'existence de deux ou plusieurs nationalités. Par conséquent, l'État national est l'État composé d'une seule nationalité et non pas d'une seule nation. En revanche, une nation peut comporter plusieurs composantes ethniques, c'est-à-dire plusieurs nationalités. Le terme nation désigne l'ensemble des nationaux d'un pays, alors que le mot nationalité vise seulement une partie de ces nationaux.

43. C'est ainsi que se dénommait un groupe d'émigrés nationalistes extrémistes.

44. Slobodan Drašković (1910-1980) participa avant la guerre à la création du Club culturel serbe dont Slobodan Jovanović fut président. Pendant la guerre, S. Drašković fut fait prisonnier par les Italiens. En 1943, il fut transféré en Allemagne dans le camp d'Osnabrück où il créa une société nationaliste et anticommuniste, appelée Dušan le Fort et qui prit, par la suite, le nom de Club culturel serbe Saint Sava. Après la guerre, il émigra aux États-Unis. Il joua un rôle de premier plan dans le schisme de l'Église serbe.

45. Extrait du compte-rendu que fit $\mathrm{S}$. Drašković devant le congrès du Club culturel serbe, cité d'après Srpska borba, 09/07/59, p. 3.

46. À un moment donné, l'idéologie se transforme en mythologie; chez les Serbes, c'est le nombre de victimes fait par l'État Indépendant de Croatie, chez les Croates, c'est le massacre commis à Bleiburg par les partisans de Tito.

47. Particulièrement le Mouvement des Tchetniks Serbes Ravna Gora dirigé par Vojvoda Đujić qui édite la revue Srbija ("Serbie") en Californie.

48. Pajić (Ivan D.), «Političko-demagoško anti jugoslovenstvo » (L'anti-yougoslavisme politicodémagogique), Naša Reč, (105), p. 105.

49. Voir chapitre «L'idée d'une Grande Croatie dans l'histoire » in Glamočak (Marina), op.cit.

50. Radić (Branko), in Nikolić (Vinko), ur., Hrvatski razgovori o slobodi (Conversations croates à propos de la liberté), München / Barcelona : Hrvatska revija, 1974, p. 316. 
51. Bušić (Bruno), « Misli prigodom 10. travnja " (Réflexions à l'occasion du 10 avril), Republika Hrvatska, (118), 1979, p. 25 (le 10 avril se réfère à la création de l'État indépendant croate en 1941).

52. Boudon (Raymond), op.cit., p. 153.

53. Ansart (Pierre), op.cit., p. 61.

54. Goati (Vladimir), Ideologija $i$ društvena stvarnost (Idéologie et réalité sociale), Beograd: Radnička štampa, 1979, p. 54.

55. Le terme "folklorique" est employé dans le sens d'une préservation de la tradition nationale.

56. Dans l'émigration politique serbe, du fait qu'existaient en Yougoslavie, lors de la Seconde Guerre mondiale, des groupes tchetniks constitués sur une base locale, les rassemblements se sont, en général, formés sur la base de l'appartenance locale et familiale et non selon des postulats politico-idéologiques.

57. Lapierre (Jean-William), «Qu'est-ce qu'une idéologie », in Amiot (M.), Borne (E.), éds., Les idéologies du monde actuel, Paris : Centre d'Études de la civilisation contemporaine, p. 15.

58. «Ainsi les crédits prévus par cette loi doivent servir à financer les activités subversives de personnes et groupements armés, activités dirigées contre l'Union Soviétique et les autres États mentionnés dans la loi.»- Archives du Quai d'Orsay, EU 1949-1955, généralités, n 51, série 33, sous-série 13, dossier 8.

59. Ibid.

60. A. Guérin donne un exemple très significatif dans son ouvrage (Guérin (A.), Commandos de la guerre froide, Genève : Édito service Euvreux, 1973), notamment chapitres II et III (pp. 76-162) où il cite le cas de Mile Rukavina. Les Américains avaient la preuve que celui-ci avait tué quatre de leurs pilotes mais cela ne les empêcha pas de le nommer à la tête d'un service qui avait pour tâche de préparer les émigrants à jouer un rôle éventuel.

61. Archives du Quai d'Orsay, EU 1940-1955, généralités, n 52, p. 160.

62. European Liberation Army ou E.L.A.

63. Archives du Quai d'Orsay, EU 1940-1955, généralités, $n^{\circ}$ 52, p. 114.

64. Archives du Quai d'Orsay, EU 1940-1950, généralités : «crédit prévu par la loi 101 : des actions subversives ", p. 114.

65. Archives du Quai d'Orsay, EU 1949-1955, généralités, n 52, p. 52.

66. Voir le texte de Stoyanovitch (V.), «Aide à Tito ou à la Yougoslavie », Yougoslav Bulletin, (1), 1953, p. 3.

67. Raketić (Z.), «Une coalition avec un objectif limité », Savremenik, (37-38), juillet-août 1959, p. 7.

68. Archives du Quai d'Orsay, EU 1940-1955, généralités, $n^{\circ} 52$, p. 111

69. Interview de Branko Pešelj (secrétaire du président du Parti paysan croate et correspondant de la revue Demokratska alternativa) in Galić (Mirko), op.cit., p. 50.

\section{RÉSUMÉS}

L'auteur étudie d'abord la composition sociale des émigrations serbes et croates de l'aprèsguerre. L'appareil répressif du régime communiste a, d'une part, empêche le retour de certains émigrés, et, d'autre part, provoqué de nouveaux départs. Elle s'intéresse ensuite aux idéologies véhiculées par les groupes émigrés, avant d'analyser comment l'émigration politique a été 
utilisée, ou, pour le cas yougoslave, les raisons de l'absence de soutien occidental. L'introduction du système multipartiste ôte à l'émigration son rôle d'opposition politique.

The author studies, first, the social composition of the post-World War II Serbian and Croatian emigration. The communist's repressive apparatus has, on one hand, prevented the return of some exiles and, on the other, provoked new departures. Then, she deals with the ideologies of the emigrating groups. She also analyses how the political emigration was used and, in the Yugoslavian case, the reasons why it hadn't been supported by the West. The introduction of multipartite system took away the role of opposition group from the emigration.

\section{AUTEUR}

\section{MARINA GLAMOČAK}

M. Glamočak est chercheur-associé au C.E.M.S. (Centre des Études et Mouvements Sociaux). 